\title{
THE BIERI-NEUMANN-STREBEL INVARIANT OF THE PURE SYMMETRIC AUTOMORPHISMS OF A RIGHT-ANGLED ARTIN GROUP
}

\author{
NIC KOBAN AND ADAM PIGGOTT
}

\begin{abstract}
We compute the BNS-invariant for the pure symmetric automorphism groups of right-angled Artin groups. We use this calculation to show that the pure symmetric automorphism group of a right-angled Artin group is itself not a right-angled Artin group provided that its defining graph contains a separating intersection of links.
\end{abstract}

\section{INTRODUCTION}

In 1987, the Bieri-Neumann-Strebel (BNS) geometric invariant $\Sigma^{1}(G)$ was introduced for a discrete group $G$. The invariant is an open subset of the character sphere $S(G)$ which carries considerable algebraic and geometric information. It determines whether or not a normal subgroup with abelian quotient is finitely generated; in particular, the commutator subgroup of $G$ is finitely generated if and only if $\Sigma^{1}(G)=S(G)$. If $M$ is a smooth compact manifold and $G=\pi_{1}(M)$, then $\Sigma^{1}(G)$ contains information on the existence of circle fibrations of $M$. Additionally, if $M$ is a 3-manifold, then $\Sigma^{1}(G)$ can be described in terms of the Thurston norm. Other aspects of the rich theory of BNS-invariant can be found in BNS87.

Although $\Sigma^{1}(G)$ has proven quite difficult to compute in general, it has been computed in the case that $G$ is a right-angled Artin group [MV95, and in the case that $G$ is the pure symmetric automorphism group of a free group OK00. In the present article we generalize the result of OK00 by computing $\Sigma^{1}(G)$ when $G$ is the pure symmetric automorphism group of a right-angled Artin group. The outcome of the computation is recorded in Theorem A to be found in $\S$ 国 below.

We also provide an application of our computation. It was shown in CRSV10, that if $A$ is the right-angled Artin group determined by a graph $\Gamma$

Date: August 15, 2018.

2010 Mathematics Subject Classification. 20F65.

Key words and phrases. right-angled Artin groups, pure symmetric automorphisms, BNS-invariant. 
that has no separating intersection of links (no SILS), then the corresponding group of pure symmetric automorphisms $P \Sigma(A)$ is itself a right-angled Artin group. We prove the converse by observing that when $\Gamma$ has a SIL, the BNS-invariant of $P \Sigma(A)$ does not have a certain distinctive property that the BNS-invariant of a right-angled Artin group must satisfy. Thus we prove:

Theorem B. The group $P \Sigma(A)$ is isomorphic to a right-angled Artin group if and only if the defining graph $\Gamma$ contains no SILs.

Theorem $\mathrm{B}$ is indicative of a dichotomy within the family of groups $\{P \Sigma(A)\}$ determined by whether or not $\Gamma$ has a SIL. Certain algebraic manifestations of this dichotomy were proved in GPR12. It would be interesting to understand more geometric manifestations. Since right-angled Artin groups are $\operatorname{CAT}(0)$ groups, we are lead to ask the following question:

Question 1.1. If the defining graph $\Gamma$ contains a SIL, is $P \Sigma(A)$ a $\operatorname{CAT}(0)$ group?

This paper is organized as follows: in $\S 2$ and $\S 3$ we define the BNSinvariant $\Sigma^{1}(G)$ and the pure symmetric automorphism group, respectively, and record some useful facts which inform the arguments to follow. We prove Theorem $\mathrm{A}$ in $\S$ 4. This proof involves two cases with the first handled in $\S 4.1$ and the second in $\S 4.2$ In $\S$, we prove Theorem B.

\section{THE BNS-INVARIANT}

Let $G$ be a finitely generated group. A character $\chi$ of $G$ is a homomorphism from $G$ to the additive reals. The set of all characters of $G$, denoted $\operatorname{Hom}(G, \mathbb{R})$, is an $n$-dimensional real vector space where $n$ is the $\mathbb{Z}$-rank of the abelianization of $G$. Two non-zero characters $\chi_{1}$ and $\chi_{2}$ are equivalent if there is a real number $r>0$ such that $\chi_{1}=r \chi_{2}$. The set of equivalence classes $S(G)=\{[\chi] \mid \chi \in \operatorname{Hom}(G, \mathbb{R})-\{0\}\}$ is called the character sphere of $G$, and this is homeomorphic to an $(n-1)$-dimensional sphere. The BNS invariant $\Sigma^{1}(G)$, a subset of $S(G)$, may be described in terms of either the geometry of Cayley graphs (see BNS87]), or $G$-actions on $\mathbb{R}$-trees (see Bro87]). For our purposes the latter is more convenient, and we now describe $\Sigma^{1}(G)$ from that point of view.

Suppose $G$ acts by isometries on an $\mathbb{R}$-tree, $T$, and let $\ell: G \rightarrow \mathbb{R}^{+}$be the corresponding length function. For each $g \in G$, let $C_{g}$ be the characteristic subtree of $g$. If $\ell(g)=0$, then $g$ is elliptic, and $C_{g}$ is its fixed point set; if $\ell(g) \neq 0$, then $g$ is hyperbolic, and $C_{g}$ is the axis of $g$. The action is nontrivial if at least one element of $G$ is hyperbolic, and abelian if every element of $[G, G]$ is elliptic. A non-trivial abelian action on an $\mathbb{R}$-tree must fix either one or two ends of the tree, and is considered exceptional if it fixes only one end. To each non-trivial abelian action, and each fixed end $e$, we associate the character $\chi$ such that $|\chi(g)|=\ell(g)$, and $\chi(g)$ is positive if and only if $g$ 
is a hyperbolic isometry which translates its axis away from the fixed end $e$. We say $g$ is $\chi$-elliptic if $\chi(g)=0$, and $\chi$-hyperbolic otherwise.

We are now able to give Brown's formulation of $\Sigma^{1}(G)$ : An equivalence class $[\chi] \in S(G)$ is contained in $\Sigma^{1}(G)$ unless there exists an $\mathbb{R}$-tree $T$ equipped with an exceptional non-trivial abelian $G$-action associated to $\chi$.

To demonstrate that $[\chi] \in \Sigma^{1}(G)$, it suffices to show that in any $\mathbb{R}$-tree $T$ equipped with a non-trivial abelian $G$-action associated to $\chi$, there exists a line $X$ such that $X \subseteq C_{g}$ for all $g \in G$. For this purpose, the following facts about characteristic subtrees are invaluable (see OK00:

Fact A: If $[g, h]=1$ and $h$ is hyperbolic, then $C_{h} \subseteq C_{g}$.

Fact B: If $[g, h]=1$, then $C_{g} \cap C_{h} \subseteq C_{g h}$.

Essentially, we work with a fixed finite generating set of $G$, we consider an arbitrary non-trivial abelian $G$-action on an arbitrary $\mathbb{R}$-tree $T$, we let $X \subseteq T$ denote the axis of one $\chi$-hyperbolic generator $s$, and we use Facts $\mathrm{A}$ and $\mathrm{B}$ to demonstrate that $X \subseteq C_{t}$ for every other generator $t$. For this approach to be successful we typically need a sufficient number of commuting relations in G.

To demonstrate that $[\chi] \in \Sigma^{1}(G)^{c}$, it is often convenient to make use of the following well-known facts.

Lemma 2.1. Let $\chi \in \operatorname{Hom}(G, \mathbb{R})-\{0\}$. Suppose there is an epimorphism $\phi: G \rightarrow H$ and a character $\psi \in \operatorname{Hom}(H, \mathbb{R})$ such that $\chi=\psi \circ \phi$. If $[\psi] \in$ $\Sigma^{1}(H)^{c}$, then $[\chi] \in \Sigma^{1}(G)^{c}$.

Corollary 2.2. If $A$ and $B$ are non-trivial finitely-generated groups, and $\chi \in \operatorname{Hom}(G, \mathbb{R})-\{0\}$ factors through an epimorphism $G \rightarrow A * B$, then $[\chi] \in \Sigma^{1}(G)^{c}$.

Proof. This follows from Lemma 2.1 and the fact that $\Sigma^{1}(A * B)=\emptyset$.

\section{Right-Angled Artin groups and their pure symmetrid AUTOMORPHISMS}

Throughout we fix a simplicial graph $\Gamma$, with vertex set $V$ and edge set $E$. For each vertex $a \in V$, the link of $a$ is the set $\operatorname{Lk}(a)=\{b \in V \mid\{a, b\} \in E\}$, and the star of $a$ is the set $\operatorname{St}(a)=\operatorname{Lk}(a) \cup\{a\}$. For a set of vertices $W \subseteq V$, we write $\Gamma \backslash W$ for the full subgraph spanned by the vertices in $V \backslash W$.

Let $A=A(\Gamma)$ denote the right-angled Artin group determined by $\Gamma$. We shall not distinguish between the vertices of $\Gamma$ and the generators of $A$, thus $A$ is the group presented by

$$
\langle V| a b=b a \text { for all } a, b \in V \text { such that }\{a, b\} \in E\rangle .
$$

For each vertex $a \in V \backslash Z$, and each connected component $K$ of $\Gamma \backslash \operatorname{St}(a)$, the map

$$
v \mapsto \begin{cases}a^{-1} v a & \text { if } v \in K, \\ v & \text { if } v \in V \backslash K,\end{cases}
$$


extends to an automorphism $\pi_{K}^{a}: A \rightarrow A$. We say $\pi_{K}^{a}$ is the partial conjugation (of $A$ ) with acting letter $a$ and domain $K$. We write $\mathcal{P}$ for the set comprising the partial conjugations.

The pure symmetric automorphism group, $P \Sigma(A)$, comprises those automorphisms $\alpha: A \rightarrow A$ which map each vertex to a conjugate of itself. Laurence proved that $P \Sigma(A)$ is generated by $\mathcal{P}$ [Lau95].

We let $Z=\{a \in V \mid \operatorname{St}(a)=V\}$, and we may assume $Z \neq \emptyset$ for the following reason: it follows immediately from Laurence's result, together with the observation that enriching $\Gamma$ with a new vertex $w$ adjacent to all other vertices does not introduce new partial conjugations, and does not change the domain of any existing partial conjugation. Let $d: V \times V \rightarrow\{0,1,2\}$ denote the combinatorial metric on $V$.

We now record three results, paraphrased from existing literature, which make working with $\mathcal{P}$ tractable. A proof of the first is included because it is so brief; the second follows immediately from the first.

Lemma 3.1. GPR12, Lemma 4.3] If $\pi_{K}^{a}, \pi_{L}^{b} \in \mathcal{P}$ and $d(a, b)=2$ and $b \notin K$, then either $K \cap L=\emptyset$ or $K \subseteq L$.

Proof. Assume $\pi_{K}^{a}, \pi_{L}^{b} \in \mathcal{P}$ and $d(a, b)=2$ and $b \notin K$. For the sake of contradiction, suppose $\emptyset \neq K \cap L \neq K$. Let $u \in K \cap L$ and $v \in K \backslash L$. Since $K$ is connected, there exists a path $\alpha$ in $K$ from $u$ to $v$. Since $u \in L$ and $v \notin L, \alpha$ passes through a vertex $w \in \operatorname{St}(b)$. Since $d(b, w) \leq 1$ and $w \in K$ and $b \in \Gamma \backslash \operatorname{St}(a), b \in K$ - contradiction.

Lemma 3.2. GPR12, Corollary 4.4 and Lemma 4.7] For each pair of partial conjugations $\left(\pi_{K}^{a}, \pi_{L}^{b}\right) \in \mathcal{P} \times \mathcal{P}$, exactly one of the following six cases holds:

(1) $d(a, b) \leq 1$;

(2) $d(a, b)=2, a \in L$, and $b \in K$;

(3) $d(a, b)=2, K \cap L=\emptyset$, and either $a \in L$ or $b \in K$;

(4) $d(a, b)=2$, and either $\{a\} \cup K \subset L$ or $\{b\} \cup L \subset K$;

(5) $d(a, b)=2$, and $(\{a\} \cup K) \cap(\{b\} \cup L)=\emptyset$;

(6) $d(a, b)=2$, and $K=L$.

The relation $\left[\pi_{K}^{a}, \pi_{L}^{b}\right]=1$ holds only in the cases (1), (4) and (5).

Theorem 3.3. Toi12, Chapter 3] Every relation between partial conjugations is a consequence of the following relations:

(1) $\left[\pi_{K}^{a}, \pi_{L}^{b}\right]=1$ if $\left(\pi_{K}^{a}, \pi_{L}^{b}\right)$ falls into one of the cases (1), (4), (5) of Lemma 3.2:

(2) $\left[\pi_{K}^{a} \pi_{L}^{a}, \pi_{L}^{b}\right]=1$ if $K \neq L$ and $b \in K$.

It is convenient to introduce notation for certain products of partial conjugations with the same acting letter. We write $\delta_{K, L}^{a}$ for the product $\pi_{K}^{a} \pi_{L}^{a}$, provided $K \neq L$. We write $\iota^{a}$ for the inner automorphism $w \mapsto a^{-1} w a$ for all 
$w \in A$, and we note $\iota^{a}$ is simply the product of all partial conjugations with acting letter $a$.

Next we record some useful facts about the behavior of partial conjugations.

Lemma 3.4. If $\pi_{K}^{a}, \pi_{L}^{b} \in \mathcal{P}$ are such that $a \notin L$ and $b \in K$ and $K \cap L=\emptyset$, then $\pi_{L}^{a} \in \mathcal{P}$ and $\left[\delta_{K, L}^{a}, \pi_{L}^{b}\right]=1$.

Proof. Assume $\pi_{K}^{a}, \pi_{L}^{b} \in \mathcal{P}$ are such that $a \notin L$ and $b \in K$ and $K \cap L=\emptyset$. Let $K^{\prime}$ denote the connected component of $\Gamma \backslash \operatorname{St}(a)$ such that $K^{\prime} \cap L \neq \emptyset$. Since $d(a, b)=2$ and $a \notin L$ and $b \notin K^{\prime}$ and $K^{\prime} \cap L \neq \emptyset$, the pair $\left(\pi_{K^{\prime}}^{a}, \pi_{L}^{b}\right)$ falls into case (6) of Lemma 3.2. Thus $K^{\prime}=L$. The relation $\left[\delta_{K, L}^{a}, \pi_{L}^{b}\right]=1$ is (2) in Theorem 3.3 .

Corollary 3.5. If $a \in V \backslash Z$ and $\pi_{L}^{b} \in \mathcal{P}$, then $\left[\iota^{a}, \pi_{L}^{b}\right]=1$ if and only if $a \notin L$.

\section{The BNS-invariant of $P \Sigma(A)$}

Throughout this section we consider an arbitrary non-trivial character $\chi$ : $P \Sigma(A) \rightarrow \mathbb{R}$. We write $\Sigma$ for $\Sigma^{1}(P \Sigma(A))$, and $\Sigma^{c}$ for the complement of $\Sigma$ in $S(P \Sigma(A))$.

Lemma 4.1. Let $\pi_{K}^{a}, \pi_{L}^{a} \in \mathcal{P}$ with $K \neq L$. If $\pi_{K}^{a}, \pi_{L}^{a}$ and $\delta_{K, L}^{a}$ are $\chi$ hyperbolic, then $[\chi] \in \Sigma$.

Proof. Suppose $\pi_{K}^{a}, \pi_{L}^{a}$ and $\delta_{K, L}^{a}$ are $\chi$-hyperbolic. Consider a $P \Sigma(A)$-action on an $\mathbb{R}$-tree $T$ that realizes $\chi$. Let $X=C_{\pi_{K}^{a}}=C_{\pi_{L}^{a}}=C_{\delta_{K, L}^{a}}$. Let $\pi_{M}^{c}$ be an arbitrary partial conjugation. If $\left[\pi_{K}^{a}, \pi_{M}^{c}\right]=1$ or $\left[\pi_{K}^{a}, \pi_{M}^{c}\right]=1$, then $X \subseteq C_{\pi_{M}^{c}}$ by Fact A; thus we may assume $\left[\pi_{K}^{a}, \pi_{M}^{c}\right] \neq 1$ and $\left[\pi_{K}^{a}, \pi_{M}^{c}\right] \neq 1$. It follows that $d(a, c)=2$. Since $K \cap L=\emptyset$, we may assume without loss of generality that $c \notin K$. Since $d(a, c)=2$ and $c \notin K$ and $\left[\pi_{K}^{a}, \pi_{M}^{c}\right] \neq 1$, the pair $\left(\pi_{K}^{a}, \pi_{M}^{c}\right)$ falls into case (3) or (6) of Lemma 3.2 .

First consider the case that $\left(\pi_{K}^{a}, \pi_{M}^{c}\right)$ falls into case (3). Then $a \in M$. By Lemma 3.4 $\pi_{K}^{c} \in \mathcal{P}$ and $\left[\delta_{K, M}^{c}, \pi_{K}^{a}\right]=1$. By Fact $\mathrm{A}, X \subseteq C_{\delta_{K, M}^{c}}$. If $c \in L$, then $\left[\delta_{K, L}^{a}, \pi_{K}^{c}\right]=1$ and $X \subseteq C_{\pi_{K}^{c}}$ by Fact A. By Fact $\mathrm{B}, X \subseteq C_{\pi_{M}^{c}}$. If $c \notin L$, then the pair $\left(\pi_{L}^{a}, \pi_{K}^{c}\right)$ falls into case (5) of Lemma 3.2 which implies $\left[\pi_{L}^{a}, \pi_{K}^{c}\right]=1$. By Fact $\mathrm{A}, X \subseteq C_{\pi_{K}^{c}}$ which implies $X \subseteq C_{\pi_{M}^{c}}$ by Fact B.

Now consider the case that $\left(\pi_{K}^{a}, \pi_{M}^{c}\right)$ falls into case (6). Then $a \notin M$, $c \notin K$ and $M=K$. Since $M \cap L=K \cap L=\emptyset$ and $a \notin M$ and $\left[\pi_{L}^{a}, \pi_{M}^{c}\right] \neq 1$, the pair $\left(\pi_{L}^{a}, \pi_{M}^{c}\right)$ falls into case (3) of Lemma 3.2. Thus $c \in L$. Since $c \in L$ and $M=K,\left[\delta_{K, L}^{a}, \pi_{M}^{c}\right]=1$, and by Fact A, $X \subseteq C_{\pi_{M}^{c}}$.

Corollary 4.2. If $[\chi] \in \Sigma^{c}$, then the following properties hold for each vertex $a \in V \backslash Z$ :

(1) There are at most two $\chi$-hyperbolic partial conjugations with acting letter $a$. 
(2) The inner automorphism $\iota^{a}$ is $\chi$-hyperbolic if and only if there is exactly one $\chi$-hyperbolic partial conjugation with acting letter $a$.

(3) If $\pi_{K}^{a}$ and $\pi_{L}^{a}$ are distinct $\chi$-hyperbolic partial conjugations, then $\chi\left(\pi_{K}^{a}\right)=$ $-\chi\left(\pi_{L}^{a}\right)$.

Lemma 4.3. Let $\pi_{K}^{a}, \pi_{L}^{a} \in \mathcal{P}$ with $K \neq L$, and let $b \in V$. If $\pi_{K}^{a}, \pi_{L}^{a}$ and $\iota^{b}$ are $\chi$-hyperbolic, then $[\chi] \in \Sigma$.

Proof. Suppose $\pi_{K}^{a}, \pi_{L}^{a}$ and $\iota^{b}$ are $\chi$-hyperbolic. If $a=b$, then $[\chi] \in \Sigma$ by Corollary 4.2(3). Thus we may assume $b \neq a$. Let $T$ be an $\mathbb{R}$-tree equipped with a $P \Sigma(A)$-action that realizes $\chi$. Let $X=C_{\pi_{K}^{a}}=C_{\pi_{L}^{a}}$. Since $\iota^{b}$ is $\chi$ hyperbolic, there exists a connected component $M$ of $\Gamma \backslash \operatorname{St}(b)$ such that $\pi_{M}^{b}$ is $\chi$-hyperbolic. If $b \notin K$, then

$$
\left[\pi_{K}^{a}, \iota^{b}\right]=\left[\iota^{b}, \pi_{M}^{b}\right]=1
$$

if $b \in K$, then $b \notin L$ and

$$
\left[\pi_{L}^{a}, \iota^{b}\right]=\left[\iota^{b}, \pi_{M}^{b}\right]=1
$$

in either case, Fact A yields

$$
C_{\pi_{L}^{a}}=C_{\iota^{b}}=C_{\pi_{M}^{b}}=X .
$$

Let $\pi_{N}^{c}$ be an arbitrary partial conjugation. The lemma is proved if we show $X \subseteq C_{\pi_{N}^{c}}$, for then the $P \Sigma(A)$-action fixes $X$ setwise and is therefore not exceptional. If $\pi_{N}^{c}$ commutes with any of the automorphisms $\pi_{K}^{a}, \pi_{L}^{a}, \pi_{M}^{b}$ or $\iota^{b}$, then $X \subseteq C_{\pi_{N}^{c}}$ by Fact A. Thus we may assume $\pi_{N}^{c}$ commutes with none of these automorphisms. It follows that $d(a, c)=d(b, c)=2$ and $b \in N$. Since $K \cap L=\emptyset$, we may assume without loss of generality that $c \notin L$. We now consider cases based on whether or not $N$ contains $a$.

First we consider the case $a \in N$. Since $b \in N$ and $c \notin L$ and $\left[\pi_{N}^{c}, \pi_{L}^{b}\right] \neq 1$, the pair $\left(\pi_{N}^{c}, \pi_{L}^{b}\right)$ falls into case (3) of Lemma 3.2, thus $N \cap L=\emptyset$. By Lemma 3.4. $\pi_{L}^{c}$ is a partial conjugation, and $\left[\pi_{L}^{a}, \delta_{L, N}^{c}\right]=1$. By Fact $\mathrm{A}, X \subseteq C_{\delta_{L, N}^{c}}$. Since $b \in N, b \notin L$, and by Corollary $3.5,\left[\iota^{b}, \pi_{L}^{c}\right]=1$. By Fact A, $X \subseteq C_{\pi_{L}^{c}}$. By Fact $\mathrm{B}, X \subseteq C_{\pi_{N}^{c}}$.

Next we consider the case $a \notin N$. Since $a \notin N$ and $c \notin L$ and $\left[\pi_{L}^{a}, \pi_{N}^{c}\right] \neq 1$, the pair $\left(\pi_{L}^{a}, \pi_{N}^{c}\right)$ falls into case (6) of Lemma 3.2. thus $N=L$. Let $N^{\prime}$ be the component of $\Gamma \backslash \operatorname{St}(c)$ such that $a \in N^{\prime}$. Therefore, $\left[\pi_{L}^{a}, \delta_{N, N^{\prime}}^{c}\right]=1$. By Fact $\mathrm{A}, X \subseteq C_{\delta_{N, N^{\prime}}^{c}}$. Since $b \in N, b \notin N^{\prime}$, and by Corollary $3.5\left[\iota^{b}, \pi_{N^{\prime}}^{c}\right]=1$. By Fact A, $X \subseteq C_{\pi_{N^{\prime}}^{c}}$. By Fact B, $X \subseteq C_{\pi_{N}^{c}}$.

Corollary 4.4. If $[\chi] \in \Sigma^{c}$, then exactly one of the following holds:

(I) For each vertex $a \in V \backslash Z$, there is at most one $\chi$-hyperbolic partial conjugation with acting letter $a$.

(II) For each vertex $a \in V \backslash Z, \iota^{a}$ is $\chi$-elliptic and there are either zero or two $\chi$-hyperbolic partial conjugations with acting letter $a$. 
Motivated by the corollary above, we classify characters depending on which case, if any, they fall into.

Definition 4.5. We say $\chi$ is type $I$ if for each vertex $a \in V \backslash Z$, there is at most one $\chi$-hyperbolic partial conjugation with acting letter $a$. We say $\chi$ is type II if for each vertex $a \in V \backslash Z, \iota^{a}$ is $\chi$-elliptic and there are either zero or two $\chi$-hyperbolic partial conjugations with acting letter $a$.

\subsection{Characters of Type I.}

Definition 4.6 (p-set). A set of partial conjugations $\mathcal{Q} \subseteq \mathcal{P}$ is a $p$-set (or a partionable set) if $\mathcal{Q}$ satisfies the following properties:

(1) For each vertex $a \in V \backslash Z, \mathcal{Q}$ contains at most one partial conjugation with acting letter $a$.

(2) The set $\mathcal{Q}$ admits a non-trivial partition $\left\{\mathcal{Q}_{1}, \mathcal{Q}_{2}\right\}$ with the property that $a \in L$ and $b \in K$ for each pair $\left(\pi_{K}^{a}, \pi_{L}^{b}\right) \in \mathcal{Q}_{1} \times \mathcal{Q}_{2}$.

We say $\left\{\mathcal{Q}_{1}, \mathcal{Q}_{2}\right\}$ is an admissible partition of $\mathcal{Q}$.

Remark 4.7. In the definition above, the first property is implied by the second. In this instance we have preferred transparency to brevity.

Remark 4.8. An arbitrary maximal p-set $\mathcal{Q}$, and an admissible partition $\left\{\mathcal{Q}_{1}, \mathcal{Q}_{2}\right\}$ may be constructed as follows. Begin with a partial conjugation $\pi_{K}^{a}$. Let $b_{1}, \ldots, b_{n}$ be the vertices of $K$. For $j=1, \ldots, n$, let $L_{j}$ be the connected component of $\Gamma \backslash \operatorname{St}\left(b_{j}\right)$ such that $a \in L_{j}$. Let $a=a_{1}, a_{2}, \ldots, a_{m}$ be the vertices of $\bigcap_{j=1}^{n} L_{j} \neq \emptyset$. For $i=1,2, \ldots, m$, let $K_{i}$ be the connected component of $\Gamma \backslash \operatorname{St}\left(a_{i}\right)$ such that $b_{1} \in K_{i}$. Let

$$
\mathcal{Q}_{1}=\left\{\pi_{K_{1}}^{a_{1}}, \ldots, \pi_{K_{m}}^{a_{m}}\right\}, \mathcal{Q}_{2}=\left\{\pi_{L_{1}}^{b_{1}}, \ldots, \pi_{L_{n}}^{b_{n}}\right\} \text {, and } \mathcal{Q}=\mathcal{Q}_{1} \cup \mathcal{Q}_{2} .
$$

Proposition 4.9. Suppose $\chi$ is type I and let $\mathcal{H}$ denote the set of $\chi$-hyperbolic partial conjugations. Then $[\chi] \in \Sigma^{c}$ if and only if $\mathcal{H}$ is contained in some $p$-set $\mathcal{Q}$.

Proof. Suppose $\chi$ is type I and $\mathcal{H}$ is contained in a p-set $\mathcal{Q}$. Let $\left\{\mathcal{Q}_{1}, \mathcal{Q}_{2}\right\}$ be an admissible partition of $\mathcal{Q}$, with

$$
\mathcal{Q}_{1}=\left\{\pi_{K_{1}}^{a_{1}}, \ldots, \pi_{K_{m}}^{a_{m}}\right\} \text { and } \mathcal{Q}_{2}=\left\{\pi_{L_{1}}^{b_{1}}, \ldots, \pi_{L_{n}}^{b_{n}}\right\} .
$$

Let $G_{1}$ be the free abelian group with basis $\left\{u_{1}, \ldots, u_{m}\right\}$, let $G_{2}$ be the free abelian group with basis $\left\{v_{1}, \ldots, v_{n}\right\}$, and let $G=G_{1} * G_{2}$. Consider a map such that: $\pi_{K_{i}}^{a_{i}} \mapsto u_{i}$ for $i=1 \ldots, m ; \pi_{L_{j}}^{b_{j}} \mapsto v_{j}$ for $j=1, \ldots, n$; and all other partial conjugations are mapped to the identity. It follows from Theorem 3.3 that this map determines an epimorphism $\phi: P \Sigma(A) \rightarrow G$. Since $\chi$ factors through $\phi$, by Corollary $2.2[\chi] \in \Sigma^{c}$.

Now suppose $\chi$ is type I and there is no p-set containing $\mathcal{H}$. Let $T$ be an $\mathbb{R}$-tree equipped with a $P \Sigma(A)$-action that realizes $\chi$. Let $\pi_{K}^{a} \in \mathcal{H}$, and let $X=C_{\pi_{K}^{a}}$. To prove the lemma it suffices to prove that $X \subseteq C_{\pi_{M}^{c}}$ for an 
arbitrary partial conjugation $\pi_{M}^{c}$, because then we have that the action fixes $X$ setwise and hence is not exceptional. If $\pi_{M}^{c}$ commutes with $\pi_{K}^{a}$, Fact A gives that $X \subseteq C_{\pi_{M}^{c}}$. Thus we may assume that $\pi_{M}^{c}$ does not commute with $\pi_{K}^{a}$.

Next we show that the elements of $\mathcal{H}$ share the axis $X$. Let

$$
\mathcal{I}=\left\{\pi_{L}^{b} \in \mathcal{H} \mid X \subseteq C_{\pi_{L}^{b}}\right\} .
$$

Suppose $\mathcal{H} \neq \mathcal{I}$, and let $\pi_{L}^{b} \in \mathcal{H} \backslash I$. Since $X \not C_{\pi_{L}^{b}}$, we have that $\left[\pi_{L}^{b}, \iota^{a}\right] \neq 1$, and $\left[\pi_{K}^{a}, \iota^{b}\right] \neq 1$. By Corollary 3.5 we have $a \in L$ and $b \in K$. It follows that $(\mathcal{I}, \mathcal{H} \backslash \mathcal{I})$ is an admissible partition, and $\mathcal{H}$ is a p-set - a contradiction which proves $\mathcal{H}=\mathcal{I}$.

Now consider an arbitrary partial conjugation such that $\pi_{M}^{c}$ does not commute with $\pi_{L}^{b}$ or $\iota^{b}$ whenever $\pi_{L}^{b} \in \mathcal{H}$. It follows that $d(b, c)=2$ for all $\pi_{K}^{b} \in \mathcal{H}$. Since $\pi_{M}^{c}$ does not commute with $\iota^{b}, b \in M$ for each $\pi_{L}^{b} \in \mathcal{H}$. Since $\mathcal{H} \cup\left\{\pi_{M}^{c}\right\}$ is not a p-set, $\left\{\left\{\pi_{M}^{c}\right\}, \mathcal{H}\right\}$ is not an admissible partition. Thus there exists $\pi_{L}^{b} \in \mathcal{H}$ such that $c \notin L$. Since $d(b, c)=2$ and $b \in M$ and $c \notin L$ and $\left[\pi_{M}^{c}, \pi_{L}^{b}\right] \neq 1$, Lemma 3.4 gives that $\pi_{L}^{c}$ is a partial conjugation. Since $\left[\delta_{L, M}^{c}, \pi_{L}^{b}\right]=1$, Fact A gives $X \subseteq C_{\delta_{L, M}^{c}}$. By Corollary $\left[3.5\right.$, $\left[\pi_{L}^{c}, \iota^{b}\right]=1$. By Fact $\mathrm{A}, X \subseteq C_{\pi_{L}^{c}}$. By Fact $\mathrm{B}, X \subseteq C_{\pi_{M}^{c}}$.

\subsection{Characters of Type II.}

Definition 4.10 ( $\delta$-p-set). A set of partial conjugations $\mathcal{Q} \subseteq \mathcal{P}$ is a $\delta$-p-set if $\mathcal{Q}$ satisfies the following properties:

(1) For each vertex $a \in V \backslash Z, \mathcal{Q}$ contains either zero or two partial conjugations with acting letter $a$.

(2) The set $\mathcal{Q}$ admits a non-trivial partition $\left\{\mathcal{Q}_{1}, \mathcal{Q}_{2}\right\}$ such that $a \in L$ or $b \in K$ or $K=L$ for each pair $\left(\pi_{K}^{a}, \pi_{L}^{b}\right) \in \mathcal{Q}_{1} \times \mathcal{Q}_{2}$.

We say $\left\{\mathcal{Q}_{1}, \mathcal{Q}_{2}\right\}$ is an admissible $\delta$-partition of $\mathcal{Q}$.

Remark 4.11. It follows from the definitions that if $\pi_{K_{1}}^{a}, \pi_{K_{-1}}^{a} \in \mathcal{Q}$ and $K_{1} \neq K_{-1}$, then either $\pi_{K_{1}}^{a}, \pi_{K_{-1}}^{a} \in \mathcal{Q}_{1}$ or $\pi_{K_{1}}^{a}, \pi_{K_{-1}}^{a} \in \mathcal{Q}_{2}$. Further, for each quadruple

$$
\left(\pi_{K_{1}}^{a}, \pi_{K_{-1}}^{a}, \pi_{L_{1}}^{b}, \pi_{L_{-1}}^{b}\right) \in \mathcal{Q}_{1} \times \mathcal{Q}_{1} \times \mathcal{Q}_{2} \times \mathcal{Q}_{2},
$$

$a \in L_{i}$ and $b \in K_{j}$ and $K_{-i}=L_{-j}$ for some $i, j \in\{-1,1\}$.

Lemma 4.12. Let $\pi_{K_{1}}^{a}, \pi_{K_{2}}^{a}, \pi_{L_{1}}^{b}, \pi_{L_{2}}^{b} \in \mathcal{P}$ be distinct partial conjugations. Then $\left[\pi_{K_{i}}^{a}, \pi_{L_{j}}^{b}\right] \neq 1$ for all $i, j \in\{1,2\}$ if and only if $\left\{\pi_{K_{1}}^{a}, \pi_{K_{2}}^{a}, \pi_{L_{1}}^{b}, \pi_{L_{2}}^{b}\right\}$ is a $\delta$-p-set.

Proof. Assume $\left[\pi_{K_{i}}^{a}, \pi_{L_{j}}^{b}\right] \neq 1$ for all $i, j \in\{1,2\}$. Without loss of generality, assume $a \notin L_{2}$ and $b \notin K_{2}$. Since $a \notin L_{2}$ and $b \notin K_{2}$ and $\left[\pi_{K_{2}}^{a}, \pi_{L_{2}}^{b}\right] \neq 1$, the pair $\left(\pi_{K_{2}}^{a}, \pi_{L_{2}}^{b}\right)$ falls into case (6) of Lemma 3.2 thus $K_{2}=L_{2}$. Since $b \notin K_{2}$ and $K_{2} \cap L_{1}=L_{2} \cap L_{1}=\emptyset$ and $\left[\pi_{K_{2}}^{a}, \pi_{L_{1}}^{b}\right] \neq 1$, the pair $\left(\pi_{K_{2}}^{a}, \pi_{L_{1}}^{b}\right)$ falls into 
case (3) of Lemma 3.2 thus $a \in L_{1}$. Since $a \notin L_{2}$ and $K_{1} \cap L_{2}=K_{1} \cap K_{2}=\emptyset$ and $\left[\pi_{K_{1}}^{a}, \pi_{L_{2}}^{b}\right] \neq 1$, the pair $\left(\pi_{K_{1}}^{a}, \pi_{L_{2}}^{b}\right)$ falls into case (3) of Lemma 3.2 thus $b \in K_{1}$. Thus $\left\{\left\{\pi_{K_{1}}^{a}, \pi_{K_{2}}^{a}\right\},\left\{\pi_{L_{1}}^{b}, \pi_{L_{2}}^{b}\right\}\right\}$ is an admissible $\delta$-partition of $\left\{\pi_{K_{1}}^{a}, \pi_{K_{2}}^{a}, \pi_{L_{1}}^{b}, \pi_{L_{2}}^{b}\right\}$. The converse follows immediately from the definitions and Lemma 3.2 ,

Lemma 4.13. Let $\pi_{K_{1}}^{a}, \pi_{K_{2}}^{a}, \pi_{M}^{c}$ be distinct partial conjugations, and let $T$ be a $\mathbb{R}$-tree equipped with a $P \Sigma(A)$-action that realizes $\chi$. If $\pi_{K_{1}}^{a}$ and $\pi_{K_{2}}^{a}$ are $\chi$-hyperbolic, $c \notin K_{1}$ and $C_{\pi_{K_{1}}^{a}} \nsubseteq C_{\pi_{M}^{c}}$, then $c \in K_{2}$ and $\pi_{K_{1}}^{c} \in \mathcal{P}$.

Proof. Suppose $\pi_{K_{1}}^{a}$ and $\pi_{K_{2}}^{a}$ are $\chi$-hyperbolic and $c \notin K_{1}$. Let $T$ be an $\mathbb{R}$-tree equipped with a $P \Sigma(A)$-action that realizes $\chi$, and suppose $C_{\pi_{K_{1}}^{a}} \nsubseteq C_{\pi_{M}^{c}}$. It follows that $d(a, c)=2$.

Since $c \notin K_{1}$ and $\left[\pi_{K_{1}}^{a}, \pi_{M}^{c}\right] \neq 1$, the pair $\left(\pi_{K_{1}}^{a}, \pi_{M}^{c}\right)$ falls into either case (3) or case (6) of Lemma 3.2 If $\left(\pi_{K_{1}}^{a}, \pi_{M}^{c}\right)$ falls into case (3), $a \in M$. By Lemma 3.4, $\pi_{K_{1}}^{c} \in \mathcal{P}$. Since $\left[\delta_{K_{1}, M}^{c}, \pi_{K_{1}}^{a}\right]=1$, but Fact B cannot be used, we must have that $\left[\pi_{K_{1}}^{c}, \pi_{K_{2}}^{a}\right] \neq 1$; thus $\left(\pi_{K_{1}}^{c}, \pi_{K_{2}}^{a}\right)$ falls into case (3) of Lemma 3.2. and $c \in K_{2}$. If $\left(\pi_{K_{1}}^{a}, \pi_{M}^{c}\right)$ falls into case (6), we have $a \notin M$ and $M=K_{1}$. But then since $a \notin M$ and $M \cap K_{2}=\emptyset$ and $\left[\pi_{K_{2}}^{a}, \pi_{M}^{c}\right] \neq 1$, the pair $\left(\pi_{K_{2}}^{a}, \pi_{M}^{c}\right)$ falls into case (3) of Lemma 3.2 Thus $c \in K_{2}$.

Proposition 4.14. Suppose $\chi$ is type II and let $\mathcal{H}$ denote the set of $\chi$ hyperbolic partial conjugations. Then $[\chi] \in \Sigma^{c}$ if and only if $\mathcal{H}$ is contained in some $\delta$-p-set $\mathcal{Q}$.

Proof. Suppose $\mathcal{H}$ is contained in some $\delta$-p-set $\mathcal{Q}$. Let $\left\{\mathcal{Q}_{1}, \mathcal{Q}_{2}\right\}$ be an admissible partition of $\mathcal{Q}$ with

$$
\mathcal{Q}_{1}=\left\{\pi_{K_{1}}^{a_{1}}, \pi_{L_{1}}^{a_{1}}, \ldots, \pi_{K_{m}}^{a_{m}}, \pi_{L_{m}}^{a_{m}}\right\} \text { and } \mathcal{Q}_{2}=\left\{\pi_{M_{1}}^{b_{1}}, \pi_{N_{1}}^{b_{1}}, \ldots, \pi_{M_{n}}^{b_{n}}, \pi_{N_{n}}^{b_{n}}\right\} .
$$

Let $G_{1}$ be the free abelian group with basis $\left\{u_{1}, \ldots, u_{m}\right\}, G_{2}$ be the free abelian group with basis $\left\{v_{1}, \ldots, v_{n}\right\}$, and $G=G_{1} * G_{2}$. Define $\phi: P \Sigma(A) \rightarrow$ $G$ by $\pi_{K_{i}}^{a_{i}} \mapsto u_{i}$ and $\pi_{L_{i}}^{a_{i}} \mapsto u_{i}^{-1}$ for $i=1, \ldots, m, \pi_{M_{j}}^{b_{j}} \mapsto v_{j}$ and $\pi_{N_{j}}^{b_{j}} \mapsto v_{j}^{-1}$ for $j=1, \ldots, n$, and all other generators map to the identity. For $\pi_{K_{i}}^{a_{i}} \in$ $\mathcal{Q}_{1}$ and $\pi_{M_{j}}^{b_{j}} \in \mathcal{Q}_{2}$, we have either $a_{i} \in M_{j}$ or $K_{i}=M_{j}$, and in either case, $\left[\pi_{K_{i}}^{a_{i}}, \pi_{M_{j}}^{b_{j}}\right] \neq 1$. Thus, $\phi$ is a well-defined epimorphism. Since $\chi$ factors through this map, by Corollary 2.2, we have $[\chi] \in \Sigma^{c}$.

Suppose $\mathcal{H}$ is not contained in some $\delta$-p-set $\mathcal{Q}$. Let $T$ be an $\mathbb{R}$-tree equipped with an $P \Sigma(A)$-action that realizes $\chi$. Since $\chi$ is type II, we have $\pi_{a, K}, \pi_{a, L} \in$ $\mathcal{H}$ for some vertex $a \in V \backslash Z$. Let $X=C_{\pi_{K}^{a}}=C_{\pi_{L}^{a}}$.

First we will show $X=C_{\pi_{M}^{b}}$ for each $\pi_{M}^{b} \in \mathcal{H}$. Define $\mathcal{I}=\left\{\pi_{M}^{b} \in \mathcal{H} \mid X=\right.$ $\left.C_{\pi_{M}^{b}}\right\}$. Assume $\mathcal{H} \neq \mathcal{I}$, and let $\pi_{M}^{b} \in \mathcal{H} \backslash \mathcal{I}$. Since $\pi_{M}^{b} \in \mathcal{H}$, there exists $\pi_{N}^{b} \in \mathcal{H}$ where $M \neq N$, and clearly $\pi_{N}^{b} \in \mathcal{H} \backslash \mathcal{I}$. Let $\pi_{Q}^{c} \in \mathcal{I}$. Again, there 
must be $\pi_{R}^{c} \in \mathcal{I}$ such that $Q \neq R$. By Lemma 4.12, $(\mathcal{I}, \mathcal{H} \backslash \mathcal{I})$ is an admissible $\delta$-partition which is a contradiction, so $\mathcal{H}=\mathcal{I}$.

Now let $\pi_{M}^{b}$ be an arbitrary element of $\mathcal{P}$, and let

$$
\mathcal{H}=\left\{\pi_{K_{1}}^{a_{1}}, \pi_{L_{1}}^{a_{1}}, \ldots, \pi_{K_{m}}^{a_{m}}, \pi_{L_{m}}^{a_{m}}\right\} .
$$

By Lemma 4.13 either $X \subseteq C_{\pi_{M}^{b}}$ or without loss of generality, $b \in K_{i}$ and $\pi_{L_{i}}^{b} \in \mathcal{P}$ for each $i=1, \ldots, m$. Assume the latter is true, so either $a_{i} \notin M$ for some $i \in\{1, \ldots, m\}$ or $a_{i} \in M$ for each $i \in\{1, \ldots, m\}$. If $a_{i} \notin M$, then $\pi_{M}^{b}$ commutes with $\pi_{L_{i}}^{a_{i}}$ which implies by Fact A that $X \subseteq C_{\pi_{M}^{b}}$. Suppose for each $i=1, \ldots, m, a_{i} \in M$. If $L_{i} \cap L_{j}=\emptyset$ for some $i \neq j$, then $\left[\pi_{L_{i}}^{b}, \pi_{L_{j}}^{a_{j}}\right]=1$ which implies $X \subseteq C_{\pi_{L_{i}}^{b}}$. Since $a \in M$ and $b \notin L_{i}$ and $L_{i} \cap M=\emptyset$, we have $\left[\delta_{L_{i}, M}^{b}, \pi_{L_{i}}^{a_{i}}\right]=1$. By Fact A, $X \subseteq C_{\delta_{L_{i}, M}^{b}}$, and by Fact B, $X \subseteq C_{\pi_{M}^{b}}$. Suppose $L_{i} \cap L_{j} \neq \emptyset$ for each pair $(i, j)$. Then $L_{i}=L_{j}$ for each pair $(i, j)$ since these are connected components of $\Gamma \backslash s t(b)$. Denote by $L$ this connected component. Then $\left(\left\{\pi_{M}^{b}, \pi_{L}^{b}\right\}, \mathcal{H}\right)$ is an admissible partition of the $\delta$-p-set $\mathcal{H} \cup\left\{\pi_{M}^{b}, \pi_{L}^{b}\right\}$ which is a contradiction. Therefore, $X \subseteq C_{\pi_{M}^{b}}$, and $[\chi] \in \Sigma$.

Proposition 4.9 and Proposition 4.14 prove our first main theorem.

Theorem A. Let $\chi: P \Sigma(A) \rightarrow \mathbb{R}$ be a character, and let $\mathcal{H}$ denote the set of $\chi$-hyperbolic partial conjugations. Then $[\chi] \in \Sigma^{c}$ if and only if $\mathcal{H}$ is contained in a set of partial conjugations $\mathcal{Q}$ such that either:

(1) The set $\mathcal{Q}$ admits a partition $\left\{\mathcal{Q}_{1}, \mathcal{Q}_{2}\right\}$ with the property that a $\in L$ and $b \in K$ for each pair $\left(\pi_{K}^{a}, \pi_{L}^{b}\right) \in \mathcal{Q}_{1} \times \mathcal{Q}_{2}$; or

(2) For each vertex $a \in V \backslash Z$, $\iota^{a}$ is $\chi$-elliptic, and $\mathcal{Q}$ contains either zero or two partial conjugations with acting letter a; and $\mathcal{Q}$ admits a partition $\left\{\mathcal{Q}_{1}, \mathcal{Q}_{2}\right\}$ with the property that $a \in L$ or $b \in K$ or $K=L$ for each pair $\left(\pi_{K}^{a}, \pi_{L}^{b}\right) \in \mathcal{Q}_{1} \times \mathcal{Q}_{2}$.

Example 4.15. Let $A=\langle a, b, c, d, e \mid[a, b],[b, c],[c, d],[c, e]\rangle$. The pure symmetric automorphism group $P \Sigma(A)$ is generated by the set

$$
\left\{\pi_{\{c, d, e\}}^{a}, \pi_{\{d\}}^{b}, \pi_{\{e\}}^{b}, \pi_{\{a\}}^{c}, \pi_{\{a, b\}}^{d}, \pi_{\{e\}}^{d}, \pi_{\{a, b\}}^{e}, \pi_{\{d\}}^{e}\right\},
$$

so $S(P \Sigma(A))$ is a 7 -dimensional sphere. The maximal p-sets are:

(1) $\mathcal{Q}_{1}=\left\{\pi_{\{c, d, e\}}^{a}, \pi_{\{a\}}^{c}, \pi_{\{a, b\}}^{d}, \pi_{\{a, b\}}^{e}\right\}$ with admissible partition $\left\{\pi_{\{c, d, e\}}^{a}\right\}$ and $\left\{\pi_{\{a\}}^{c}, \pi_{\{a, b\}}^{d}, \pi_{\{a, b\}}^{e}\right\}$,

(2) $\mathcal{Q}_{2}=\left\{\pi_{\{c, d, e\}}^{a}, \pi_{\{d\}}^{b}, \pi_{\{a, b\}}^{d}\right\}$ with admissible partition $\left\{\pi_{\{c, d, e\}}^{a}, \pi_{\{d\}}^{b}\right\}$ and $\left\{\pi_{\{a, b\}}^{d}\right\}$,

(3) $\mathcal{Q}_{3}=\left\{\pi_{\{c, d, e\}}^{a}, \pi_{\{e\}}^{b}, \pi_{\{a, b\}}^{e}\right\}$ with admissible partition $\left\{\pi_{\{c, d, e\}}^{a}, \pi_{\{e\}}^{b}\right\}$ and $\left\{\pi_{\{a, b\}}^{e}\right\}$, and

(4) $\mathcal{Q}_{4}=\left\{\pi_{\{e\}}^{d}, \pi_{\{d\}}^{e}\right\}$ 
The only maximal $\delta$-p-set is $\left\{\pi_{\{d\}}^{b}, \pi_{\{e\}}^{b}, \pi_{\{a, b\}}^{d}, \pi_{\{e\}}^{d}, \pi_{\{a, b\}}^{e}, \pi_{\{d\}}^{e}\right\}$ with admissible partition $\left\{\pi_{\{d\}}^{b}, \pi_{\{e\}}^{b}\right\}$ and $\left\{\pi_{\{a, b\}}^{d}, \pi_{\{e\}}^{d}, \pi_{\{a, b\}}^{e}, \pi_{\{d\}}^{e}\right\}$. Therefore, $\Sigma^{c}$ consists of the characters $[\chi]$ such that:

(1) $\chi$ sends all generators to zero except maybe those generators in $\mathcal{Q}_{i}$ for some $1 \leq i \leq 4$, or

(2) $\chi\left(\pi_{\{d\}}^{b}\right)=-\left(\pi_{\{e\}}^{b}\right), \chi\left(\pi_{\{a, b\}}^{d}\right)=-\chi\left(\pi_{\{e\}}^{d}\right), \chi\left(\pi_{\{a, b\}}^{e}\right)=-\chi\left(\pi_{\{d\}}^{e}\right)$, and $\chi$ sends all other generators to zero.

\section{Right-Angled Artin groups with SeParating intersecting Links}

A graph $\Gamma$ has a separating intersection of links (SIL) if there exists a pair $a, b$ of distinct non-adjacent vertices such that $\Gamma \backslash(\operatorname{Lk}(a) \cap \operatorname{Lk}(b))$ has a connected component $M$ containing neither $a$ nor $b$. The following proposition was proven in CRSV10, and we state the result in terms of our particular circumstance.

Proposition 5.1. CRSV10, Theorem 3.6] If the defining graph $\Gamma$ contains no SILs, then $P \Sigma(A)$ is isomorphic to a right-angled Artin group.

In this section we prove the converse to Proposition [5.1, which completes the proof of Theorem B. We continue to use the notation described above.

Given a non-trivial character $\psi: A \rightarrow \mathbb{R}$, we write $\Gamma_{\psi}$ for the full subgraph of $\Gamma$ spanned by the set of $\psi$-hyperbolic vertices. The subgraph $\Gamma_{\psi}$ is called dominating if every vertex in $\Gamma$ is either in, or adjacent to a vertex in, $\Gamma_{\psi}$. It was shown in MV95] that:

Theorem 5.2. MV95, Theorem 4.1] Suppose $[\psi] \in S(A)$. Then $[\psi] \in \Sigma^{1}(A)$ if and only if $\Gamma_{\psi}$ is connected and dominating.

For each set of vertices $U \subseteq V$, we write $S(U)$ for the sub-sphere

$$
\{[\psi] \in S(A) \mid \psi(v)=0 \text { for all } v \in V \backslash U\} .
$$

We note that $S(U)$ is a sub-sphere of dimension $|U|-1$ (we consider $S(\emptyset)$ to be a sub-sphere of dimension -1 ). We say $S(U)$ is a missing sub-sphere if $S(U) \subseteq \Sigma(A)^{c}$, and we note this holds exactly when the full subgraph spanned by $U$ is disconnected or non-dominating. If $U$ spans a subgraph of $\Gamma$ which is non-dominating, then every subset of $U$ spans a subset of $\Gamma$ which is non-dominating; if $U$ spans a subgraph of $\Gamma$ which is disconnected, then every subset of $U$ spans a subset of $\Gamma$ which is disconnected or non-dominating. It follows that if $S(U)$ and $S(W)$ are missing sub-spheres, then $S(U \cap W)$ is a missing sub-sphere. It also follows that $\Sigma^{1}(A)$ is constructed from $S(A)$ by removing the maximal missing sub-spheres. Viewing the construction of $\Sigma^{1}(A)$ in this distinctive way, we observe the following: 
Lemma 5.3. If $A$ is a right-angled Artin group, and $S_{1}, \ldots, S_{p} \subseteq S(A)$ are the maximal missing sub-spheres, then

$$
\begin{aligned}
\operatorname{rk}(A /[A, A]) & -\operatorname{rk}(Z(A))=1+\sum_{i} \operatorname{dim}\left(S_{i}\right)-\sum_{i<j} \operatorname{dim}\left(S_{i} \cap S_{j}\right) \\
& +\sum_{i<j<k} \operatorname{dim}\left(S_{i} \cap S_{j} \cap S_{k}\right)-\cdots+(-1)^{n-1} \operatorname{dim}\left(S_{1} \cap \cdots \cap S_{p}\right) .
\end{aligned}
$$

Proof. Since $\operatorname{rk}(A /[A, A])=|V|$, and $\operatorname{rk}(Z(A))=|Z|$, the lemma is proved if we show that the right-hand side of the equation sums to $|V \backslash Z|$. It follows from Theorem 5.2 that, for each $i, S_{i}=S\left(U_{i}\right)$ for some maximal set of vertices $U_{i}$ which spans a disconnected or non-dominating subgraph of $\Gamma$. For each vertex $v \in V \backslash Z$, the singleton set $\{v\}$ spans a non-dominating subgraph of $\Gamma$, and hence $v$ is contained in at least one set $U_{i}$. Any set of vertices containing an element of $Z$ spans a connected and dominating subgraph of $\Gamma$. Thus we have $V \backslash Z=U_{1} \cup U_{2} \cup \cdots \cup U_{p}$. Now the Principle of Inclusion-Exclusion, together with the identity $\sum_{i=1}^{p}(-1)^{i-1}\left(\begin{array}{c}p \\ i\end{array}\right)=1$, gives:

$$
\begin{aligned}
& \left|U_{1} \cup U_{2} \cup \cdots \cup U_{p}\right| \\
= & \sum_{i}\left|U_{i}\right|-\sum_{i<j}\left|U_{i} \cap U_{j}\right|+\sum_{i<j<k}\left|U_{i} \cap U_{j} \cap U_{k}\right|-\ldots \\
& \ldots+(-1)^{n-1}\left|U_{1} \cap \cdots \cap U_{p}\right| \\
= & \sum_{i}\left(\operatorname{dim}\left(S_{i}\right)+1\right)-\sum_{i<j}\left(\operatorname{dim}\left(S_{i} \cap S_{j}\right)+1\right) \\
& +\sum_{i<j<k}\left(\operatorname{dim}\left(S_{i} \cap S_{j} \cap S_{k}\right)+1\right)-\ldots \\
& \cdots+(-1)^{n-1}\left(\operatorname{dim}\left(S_{1} \cap \cdots \cap S_{p}\right)+1\right) \\
= & 1+\sum_{i} \operatorname{dim}\left(S_{i}\right)-\sum_{i<j} \operatorname{dim}\left(S_{i} \cap S_{j}\right) \\
& +\sum_{i<j<k} \operatorname{dim}\left(S_{i} \cap S_{j} \cap S_{k}\right)-\cdots+(-1)^{n-1} \operatorname{dim}\left(S_{1} \cap \cdots \cap S_{p}\right) .
\end{aligned}
$$

Next we characterize the maximal missing sub-spheres in $S(A)$ by a property which makes no reference to the canonical generating set of $A$, thereby allowing us to identify the only candidates for maximal missing sub-spheres in $S(G)$ when we do not yet know whether or not $G$ is a right-angled Artin group. 
A normal subgroup $K$ in a finitely-generated group $G$ is a complement kernel if $K=\operatorname{ker}(\psi)$ for some $[\psi] \in \Sigma(G)^{c}$. For such $K$, the set

$$
\left\{[\psi] \in \Sigma^{1}(G)^{c} \mid K \subseteq \operatorname{ker}(\psi)\right\}
$$

is the complement subspace determined by $K$.

Lemma 5.4. For each subset $S \subseteq S(A), S$ is a maximal missing sub-sphere if and only if $S$ is the complement subspace determined by some minimal complement kernel $K$.

Proof. Suppose $S=S(U)$ is a maximal missing sub-sphere in $S(A)$, with $U=\left\{u_{1}, \ldots, u_{p}\right\}$. Let $\psi_{U}: A \rightarrow \mathbb{R}$ denote the character such that

$$
\psi_{U}(v)=0 \text { for } v \in V \backslash U \text {, and } \psi_{U}\left(u_{i}\right)=\pi^{i} \text { for } i=1, \ldots, p \text {. }
$$

Since $\pi$ is transcendental, $K_{U}=\operatorname{ker}\left(\psi_{U}\right)$ consists of those elements $a \in A$ with zero exponent sums in each of the vertices $u_{1}, \ldots, u_{p}$. It follows that $\left[\psi_{U}\right] \in S(U)$, and $K_{U} \subseteq \operatorname{ker}(\psi)$ for every $[\psi] \in S(U)$. Thus $S(U)$ is the complement subspace determined by $K_{U}$. The maximality of $U$, together with Theorem [5.2. implies that $K_{U}$ is minimal amongst the kernels of characters in $\Sigma^{1}(A)^{c}$. It also follows from Theorem 5.2 that every minimal complement kernel arises in this way.

We now have an approach for showing that a finitely-generated torsion-free group $G$ is not a right-angled Artin group: we identify the minimal complement kernels $K_{1}, \ldots, K_{p}$ in $G$; use these to identify the corresponding complement subspaces $S_{1}, \ldots, S_{p}$ in $S(G)$; then show that Lemma 5.3 fails. We carry out this plan for $P \Sigma(A)$ when $\Gamma$ contains a SIL.

Lemma 5.5. If $S$ is the complement subspace corresponding to a minimal complement kernel $K$ in $P \Sigma(A)$, then either:

$$
S=\left\{[\chi] \in S(P \Sigma(A)) \mid \chi\left(\pi_{K}^{a}\right)=0 \text { for all } \pi_{K}^{a} \in \mathcal{P} \backslash \mathcal{Q}\right\}
$$

for some maximal $p$-set $\mathcal{Q}$, in which case $\operatorname{dim}(S)=|\mathcal{Q}|-1$; or

$S=\left\{[\chi] \in S(A) \mid \chi\left(\pi_{K}^{a}\right)=0\right.$ for all $\pi_{K}^{a} \in \mathcal{P} \backslash \mathcal{Q}$, and $\chi\left(\iota^{v}\right)=0$ for all $\left.v \in V\right\}$ for some maximal $\delta$-p-set $\mathcal{Q}$, in which case $\operatorname{dim}(S)=|\mathcal{Q}| / 2-1$.

Proof. Suppose $S$ is the complement subspace corresponding to a minimal complement kernel $K$ in $P \Sigma(A)$, and let $\chi: P \Sigma(A) \rightarrow \mathbb{R}$ be a character with kernel $K$. By Corollary 4.4 $\chi$ is type I or type II.

Consider first the case that $\chi$ is type I. By Proposition 4.9, the $\chi$-hyperbolic vertices comprise a p-set $\mathcal{Q}$. The minimality of $K$ implies that $\mathcal{Q}$ is not contained in a larger p-set. That $S$ is as described follows immediately.

Now consider the case that $\chi$ is type II. By Proposition 4.14, the $\chi$ hyperbolic vertices comprise a $\delta$-p-set $\mathcal{Q}$. The minimality of $K$ implies that $\mathcal{Q}$ is not contained in a larger $\delta$-p-set. That $S$ is as described follows immediately. 
Lemma 5.6. If $\mathcal{Q}_{1}, \ldots, \mathcal{Q}_{p}$ are the maximal p-sets in $P \Sigma(A)$, and $S_{1}, \ldots, S_{p}$ the corresponding complement subspaces, then

$$
\begin{aligned}
\operatorname{rk}(P \Sigma(A) /[ & P \Sigma(A), P \Sigma(A)])=1+\sum_{i} \operatorname{dim}\left(S_{i}\right)-\sum_{i<j} \operatorname{dim}\left(S_{i} \cap S_{j}\right) \\
& +\sum_{i<j<k} \operatorname{dim}\left(S_{i} \cap S_{j} \cap S_{k}\right)-\cdots+(-1)^{n-1} \operatorname{dim}\left(S_{1} \cap \cdots \cap S_{p}\right) .
\end{aligned}
$$

Proof. It follows from Theorem 3.3 that $\operatorname{rk}(P \Sigma(A) /[P \Sigma(A), P \Sigma(A)])=|\mathcal{P}|$. Suppose $\pi_{K}^{a} \in \mathcal{P}$. Let $b$ be a vertex in $K$, and let $L$ be the connected component of $\Gamma \backslash \operatorname{St}(b)$ such that $a \in L$. Then $\left\{\pi_{K}^{a}, \pi_{L}^{b}\right\}$ is a p-set. Thus every partial conjugation is contained in at least one p-set. Now, as in the proof of Lemma 5.3 the lemma follows from the Principle of Inclusion-Exclusion and the identity $\sum_{i=1}^{p}(-1)^{i-1}\left(\begin{array}{l}p \\ i\end{array}\right)=1$.

Corollary 5.7. If $P \Sigma(A)$ is isomorphic to a right-angled Artin group, then $\Sigma^{1}(P \Sigma(A))^{c}$ contains no characters of type II.

Proof. Suppose $P \Sigma(A)$ is isomorphic to a right-angled Artin group, and assume the notation of Lemma 5.6. It follows from Theorem 5.3 and Lemma 5.6. that $S_{1}, \ldots, S_{p}$ is the complete list of complement subspaces corresponding to minimal complement kernels (and $P \Sigma(A)$ has no center). Thus $S_{1}, \ldots, S_{p}$ is the complete list of maximal missing sub-spheres in $S(P \Sigma(A))$, and

$$
\Sigma^{1}(P \Sigma(A))^{c}=\bigcup_{i=1}^{p} S_{i} .
$$

Since each character in each $S_{i}$ is type I, and by Corollary 4.4 no character is type I and type II, we conclude that $\Sigma^{1}(P \Sigma(A))^{c}$ contains no characters of type II.

Proposition 5.8. If $\Gamma$ contains a SIL, then $P \Sigma(A)$ is not isomorphic to a right-angled Artin group.

Proof. Suppose $\Gamma$ contains a SIL. Let $a, b$ and $M$ be as in the definition of a SIL, let $K$ be the connected component of $\Gamma \backslash \operatorname{St}(a)$ that contains $b$, and let $L$ be the connected component of $\Gamma \backslash \operatorname{St}(b)$ that contains $a$. The set $\left\{\pi_{K}^{a}, \pi_{M}^{a}, \pi_{L}^{b}, \pi_{M}^{b}\right\}$ is a $\delta$-p-set. In particular, $\Sigma^{1}(P \Sigma(A))$ contains at least one character of type II. By Corollary [5.7 $P \Sigma(A)$ is not isomorphic to a right-angled Artin group.

Proposition [5.8 and [CRSV10, Theorem 3.6] prove Theorem B.

\section{REFERENCES}

[BNS87] Robert Bieri, Walter D Neumann, and Ralph Strebel, A geometric invariant of discrete groups, Inventiones mathematicae 90 (1987), no. 3, 451-477. 
[Bro87] Kenneth S Brown, Trees, valuations, and the bieri-neumann-strebel invariant, Inventiones mathematicae 90 (1987), no. 3, 479-504.

[CRSV10] Ruth Charney, Kim Ruane, Nathaniel Stambaugh, and Anna Vijayan, The automorphism group of a graph product with no sil, Illinois Journal of Mathematics 54 (2010), no. 1, 249-262.

[GPR12] Mauricio Gutierrez, Adam Piggott, and Kim Ruane, On the automorphisms of a graph product of abelian groups, Groups, Geometry, and Dynamics 6 (2012), no. $1,125-153$.

[Lau95] Michael R. Laurence, A generating set for the automorphism group of a graph group, J. London Math. Soc. (2) 52 (1995), no. 2, 318-334. MR 1356145 (96k:20068)

[MV95] John Meier and Leonard VanWyk, The bieri-neumann-strebel invariants for graph groups, Proceedings of the London Mathematical Society 3 (1995), no. 2, 263-280.

[OK00] Lisa Orlandi-Korner, The bieri-neumann-strebel invariant for basis-conjugating automorphisms of free groups, Proceedings of the American Mathematical Society 128 (2000), no. 5, 1257-1262.

[Toi12] Emmanuel Toinet, Automorphismes des groupes d'artin à angles droits, Ph.D. thesis, Université de Bourgogne, 2012.

Dept. of Math., University of Maine Farmington, Farmington ME 04938

E-mail address: nicholas.koban@maine.edu

Dept. of Math., Bucknell University, Lewisburg PA 17837

E-mail address: adam.piggott@bucknell.edu 